\title{
CST interacts with the cohesin complex and promotes chromosome cohesion
}

\author{
P. Logan Schuck and Jason A. Stewart*
}

Department of Biological Sciences, University of South Carolina, Columbia, SC, USA

*Corresponding author: jason.stewart@sc.edu

\section{Short title: CST promotes chromosome cohesion}

Keywords: Chromosome cohesion, cohesin, CST, DNA replication, CTC1, STN1, TEN1, fork stalling

\begin{abstract}
Sister chromatid cohesion (SCC) is established during DNA replication by loading of the cohesin complex on newly replicated chromatids. Cohesin must then be maintained until mitosis to prevent segregation defects and aneuploidy. How SCC is established and maintained until mitosis remains incompletely understood and emerging evidence suggests that replication stress can lead to premature SCC loss. Here, we report that the single-stranded DNA-binding protein CTC1-STN1-TEN1 (CST) aids in SCC. CST primarily functions in telomere length regulation but also has known roles in replication restart and DNA repair. Following depletion of CST subunits, we observed an increase in the complete loss of SCC. Additionally, we determined that CST interacts with the cohesin complex. Unexpectedly, we did not find evidence of defective cohesion establishment or mitotic progression in the absence of CST. However, we did find that treatment with various replication inhibitors increased the association between CST and cohesin. Since replication stress was recently shown to induce SCC loss, we supposed that CST may be required to maintain SCC following fork stalling. In agreement with this idea, SCC loss was greatly increased in CST-depleted cells following exogenous replication stress. Based on our findings, we propose that CST aids in the maintenance of SCC at stalled replication forks to prevent premature cohesion loss.
\end{abstract}

\section{Introduction}

As DNA is replicated, the sister chromatids must be held together until mitosis to ensure chromosomes are properly segregated between daughter cells. This process, known as sister chromatid cohesion (SCC), is facilitated by the cohesin complex $(1,2)$. In mammals, the cohesin complex is 
composed of a ring-like structure that encircles the DNA. The core structure is composed of SMC3, SMC1A, SCC1 (also known was RAD21) and SA1 or SA2. Cohesin loading and removal is tightly regulated during the cell cycle (3-5). In G1 and early S-phase, cohesin is loaded onto chromatin but is not stably bound. As the DNA is replicated, cohesin is passaged to the replicated sister chromatids and becomes stably bound until mitosis when it is removed to allow segregation of the chromatids into daughter cells. In addition to its essential role in SCC, the cohesin complex is involved in organizing topologically associated domains for cellular processes such as DNA repair and gene expression (6). Cohesin has also been implicated in replication fork restart with perturbations in cohesion dynamics following fork stalling promoting premature SCC loss (7-9).

In this study, we present unexpected findings that human CTC1-STN1-TEN1 (CST) helps to maintain SCC. CST is an RPA-like, sSDNA binding protein that is conserved from yeast to humans (10). Like RPA, CST contains multiple oligosaccharide-oligonucleotide folds (OB-folds) that allow dynamic binding to the DNA $(11,12)$. CST primarily functions in telomere length regulation, however, it has also been shown to function in DNA replication and repair $(13,14)$. While its role in DNA replication is still not well understood, it is proposed to aid in the replication of G-rich DNA sequences, such as telomeres, promote dormant origin firing and negatively regulate origin licensing (15-18). CST also interacts with several components of the replication machinery, including DNA polymerase $\alpha$-primase, the MCM2-7 helicase and AND-1/Ctf4 $(16,19,20)$. Together, these findings provide strong evidence that CST is involved in DNA replication, presumably as a specialized versus general replication factor.

Here, we report that depletion of CST leads to premature SCC loss. Furthermore, we show that CST interacts with the cohesin subunits, SMC1A and SMC3, suggesting that CST may directly influence SCC. Upon further investigation, we found that the interaction between cohesin and CST is increased following replication stress and that CST prevents SCC loss following treatment with several replication inhibitors. Together, our findings identify CST as a new factor involved in preventing premature cohesion loss and suggest that it does so by stabilizing chromosome cohesion following replication fork stalling.

\section{Results}

\section{Depletion of CST results in sister chromatid cohesion loss}

While performing telomere-FISH in STN1 knockdown cells, we consistently observed metaphase spreads that had lost SCC. To determine whether depletion of STN1 increased SCC loss, we quantified the number of metaphase spreads with SCC loss in HeLa cells with stable shRNA knockdown of STN1 (shSTN1) (Fig 1A-B) (18). In agreement with our observation, we observed a 2- to 4-fold increase in premature SCC loss in two separate shSTN1 clones, shSTN1-6 and shSTN1-7 (Fig 1C). Furthermore, this increase was largely rescued by stable expression of a Flag-tagged shRNA-resistant STN1 construct in shSTN1-7 cells (shSTN1-7 +Flag-STN1). Previous studies suggest that a common off-target effect of RNA interference is MAD2 depletion (21). Loss of MAD2 also results in premature SCC loss. MAD2 levels 
were measured in the shSTN1 cells and no significant changes were observed compared to controls (Fig. 1A).

To confirm our findings, we next performed mitotic shake-off to measure SCC loss following STN1 depletion. Processing of cells for standard metaphase spread analysis includes treatment with a hypotonic solution, which can release proteins from the chromatin (22). If cohesion is only partially lost, this can exacerbate the number of cells with SCC loss. To address this possibility, mitotic cells were collected, fixed and spun onto slides without hypotonic treatment. Unlike standard metaphase spread preparation, cytospun metaphase cells are not clearly separated. Therefore, a chromosome specific probe to centromere six was used to assess SCC loss by fluorescence in situ hybribization, as previously described (23). Since HeLa cells are triploid for chromosome six, three spots indicate the retention of SCC, whereas greater than three spots indicate SCC loss. Consistent with metaphase spread analysis, knockdown of STN1 lead to a significant increase in SCC loss (Fig. 1D-E). However, the fold increase was less than that observed by standard metaphase spread analysis (Fig. 1C). This may be due to weakened but not complete SCC loss in a subset of cells or differences between the methodologies used.

Next, we determined whether cohesion loss was specific to STN1 depletion or due to a general loss of CST. siRNA knockdown of CTC1, STN1 or TEN1 was performed followed by metaphase spread analysis (Fig 1F-G). Like shSTN1 cells, transient siRNA knockdown of individual CST subunits resulted in increased SCC loss. Finally, we confirmed that this phenotype was not cell type specific by measuring SCC loss in HCT116 cells with conditional CTC1 knockout, which also showed increased SCC loss (Fig. S1). Together, these results indicate that CST promotes SCC.

\section{CST interacts with the cohesin complex}

We next addressed whether CST interacts with the cohesin complex by co-immunoprecipitation (co-IP) and proximity ligation assay (PLA) (Fig 2). IP of epitope-tagged CST pulled down both endogenous SMC3 and SMC1A (Fig 2A). STN1 alone also showed interaction with SMC3, although at lower levels. Pulldown of CTC1 showed little to no association with SMC3 or SMC1A. This suggests that the entire CST complex, or at least multiple subunits, may be required for stable interaction. As further confirmation of CST-cohesin association, we identified both SMC1A and SMC3 by mass spectrometry after IP of Flag-STN1 in cells overexpressing all three CST subunits (Fig S2).

To determine whether this interaction was also present in cells, PLA was performed using antibodies to endogenous STN1 and SMC3 (Figure 2B). PLA detects interactions through use of secondary antibodies with a segment of DNA attached. When these antibodies are in close proximity $(<40 \mathrm{~nm})$ the DNA segments are joined and the DNA is then amplified by rolling circle amplification and detected using fluorescent probes (24). PLA analysis with antibodies to endogenous STN1 and SMC3 showed $\sim 6$ foci on average compared to single antibodies controls (Fig 2B). Combined with the co-IP data, these findings reveal an unanticipated interaction between CST and the cohesin complex. 


\section{CST is not required for SMC3 acetylation, cohesin loading or mitotic progression}

A possible explanation for SCC loss following CST depletion is that CST stabilizes cohesin loading or establishment $(25,26)$. Prior to genome duplication, cohesin binding is unstable and cohesin molecules quickly associate/dissociate from the DNA (27). As the genome is duplicated, cohesin is transferred from the unreplicated to the replicated sister chromatids and becomes stably bound. This process is partially facilitated by the acetylation of SMC3 (Ac-SMC3) (28-31). Since CST aids in DNA replication, we tested whether Ac-SMC3 or the levels of total or chromatin-bound cohesin were decreased in STN1-depleted cells (Fig. 3A-C, S3A-B). However, we did not observe any changes in either Ac-SMC3 or chromatin-bound cohesin.

Since our data suggest that cohesion establishment is unaffected by STN1 depletion, we investigated whether mitotic progression was altered using live cell imaging (Fig 3D-E). To visualize mitotic events, an H2B construct fused with red fluorescence protein was stably transduced into STN1depleted or control cells (32). Cells were plated and imaged at $5 \mathrm{~min}$ intervals over a 3-hour period. The time from prophase to the completion of cytokinesis was then measured as a readout of mitotic progression (Figure 3D). Mitosis took approximately one hour in the HeLa cells, consistent with previous studies (33). On average, shSTN1 cells took $\sim 5$ minutes less to complete mitosis compared to control cells (Fig. 3E). Further breakdown of the timing from prophase to metaphase or metaphase to cytokinesis did not reveal any significant changes (Fig S3C). While there was a slight decrease in overall mitotic progression in shSTN1 cells, these changes are unlikely to explain the increase in SCC loss. Why premature loss of SCC does not lead to defects in mitotic progression in cells lacking CST is not entirely clear. However, work in Drosophila suggest that premature SCC loss is not always sufficient to trigger a robust spindle assembly checkpoint and that mitosis can still occur $(34,35)$. Moreover, recent work showed that various cancer cell lines continue to grow despite significant levels of cohesion loss $(8,26)$. Accordingly, mitotic progression and cell division may be unaffected despite increased SCC loss in STN1 depleted cells.

\section{CST promotes SCC following replication stress}

Since CST aids in DNA replication restart and SCC loss has been linked to replication stress, we sought to determine whether chemically induced replication stress would increase the interaction between CST and cohesin. Cells were treated with hydroxyurea (HU), aphidicolin (APH) or camptothecin (CPT). PLA was then performed with antibodies to endogenous STN1 and SMC3 (Fig. 4A). In all cases, we observed an $\sim 2$-fold increase in PLA foci, indicating that cohesin-CST interaction is increased following replication stress compared to untreated cells. This suggested to us that CST may prevent SCC loss following replication stress, so we tested whether SCC loss was increased after treatment with replication inhibitors in shSTN1 cells. Following treatment with replication inhibitors, cells were released into fresh media for 6 hours to allow cells in S-phase to reach mitosis. They were then treated with 
colcemid, collected and prepared for metaphase spread analysis (Fig 4B). In agreement with previous findings, SCC loss was increased in the control cells when treated with the replication inhibitors $(8,9)$. (CPT likely has the greatest effect because it is not reversible like HU and APH.) However, in the STN1 depleted cells, premature SCC loss was greatly increased above the shNT cells, consistent with CST promoting SCC following replication stress.

\section{Discussion}

In this study, we present data that CST is involved in SCC and interacts with the cohesin complex. Despite significant levels of SCC loss in CST depleted cells, we were unable to detect any defects in cohesion establishment or mitotic progression. Instead, CST appears to be involved in SCC maintenance/re-establishment at stalled replication forks. This idea is in line with recent work demonstrating that replication stress, caused by expression of oncogenes or treatment with DNA replication inhibitors, induces SCC loss $(8,9)$. Based on our data, we propose that CST acts at stalled replication forks to maintain or re-establish SCC following fork restart (Fig 4C). These findings highlight an unexpected function of CST in preserving genome integrity through the maintenance of SCC.

Over a decade ago, depletion of the replication fork stability factors Tipin/Tim and AND1 were shown to increase SCC loss, indirectly associating replication defects with chromatid cohesion (36-39). Additional studies have implicated other DNA replication and repair factors in chromatid cohesion and this past year a pair of studies provided direct evidence that chemically induced replication stress leads to SCC loss $(8,9)$. These recent studies both suggest that the cohesin antagonist WAPL is involved in cohesion maintenance following replication stress. Benedict et al. propose a model where WAPL removes cohesin to allow replication fork restart through RAD51-dependent mechanisms. However, other studies, in both budding yeast and humans, suggest that cohesin may be necessary for fork restart or protection $(7,40,41)$. Regardless of the mechanism, a picture is emerging where local cohesin architecture may be remodeled at stalled replication forks to allow restart. Interestingly, CST is proposed to load RAD51 following fork stalling (17). Therefore, CST could be a key player in cohesin dynamics at stalled forks by facilitating fork restart and cohesion reestablishment.

Another potential link between CST and cohesion maintenance is that CST interacts with both MCM2-7 and AND-1 (16). Recent work identified interaction between the MCM2-7 helicase and ESCO2 as necessary for cohesion establishment $(25,28,31)$. As mentioned above, AND-1 is involved in replication fork stability and helps maintain SCC (38). Studies in yeast suggest that AND-1 (known as $\mathrm{Ctf} 4$ in yeast) stabilizes critical interactions between replisome components and the Chl1 helicase, which is involved in chromatid cohesion $(42,43)$. We previously showed that loss of CST leads to decreased chromatin-bound AND-1, which could also provide a potential explanation for increased SCC loss in the absence of CST. We propose that interactions between CST, MCM2-7 and AND-1 are necessary for 
CST to help stabilize cohesion at stalled forks. Future studies will investigate how these interactions affect chromosome cohesion and fork restart/protection following fork stalling.

While the function of CST in replication restart/rescue is still unclear, CST is not a general replication factor but rather plays a specialized role in facilitating replication through G-rich regions of the genome (e.g. telomeres, CpG islands) $(15,17,18)$. Since CST promotes replication at specific sites, one might predict that the absence of CST would cause partial versus total loss of chromosome cohesion, as has been previously observed with depletion of the cohesin subunits SA1 or SA2 (23). Instead, CST depletion leads to complete SCC loss by metaphase spread analysis and mitotic shake-off (Figure 1). These findings demonstrate that SCC loss is not restricted to telomeres or specific regions of the genome but instead a complete breakdown of SCC. The reason for complete cohesion loss remains unclear. However, recent studies suggest that complete cohesion loss is common across a variety of cancer cells, which seems to have little effect on cellular division (8). It is possible that the gradual accumulation of SCC loss due to replication stress or excessive DNA damage, triggers genome-wide cohesin unloading through an unknown mechanism. Perhaps such a pathway is used to induce cell death to prevent the propagation of cells with high levels of genome instability induced by replication stress. In cancer, such pathways could be disengaged and allow cell division and aneuploidy, despite SCC loss. Future studies will be needed to fully investigate the connection between replication stress, SCC and aneuploidy.

\section{Experimental procedures}

\section{Cell culture}

HeLa 1.2.11 cells were cultured in RPMI 1640 media, HEK293T in DMEM, and HCT116 in McCoy's 5A media at $37^{\circ} \mathrm{C}$ with $5 \% \mathrm{CO}_{2}$. All media was supplemented with $10 \%$ fetal bovine serum and $1 \%$ penicillin/streptomycin. Stable HeLa 1.2.11 shRNA knockdown and HCT116 CTC1 inducible knockout lines have been previously described $(18,44)$. Cell lines were regularly checked for mycoplasma contamination. siRNA experiments were performed using, $25 \mathrm{nM}$ ON-TARGETplus siRNA SMART pools (Dharmacon) to CTC1 (L-014585-01), STN1 (L-016208-02), TEN1 (L-187549-00), or Non-targeting control (D-00180-10). siRNAs were transfected into cells with Lipofectamine RNAiMAX (Thermo Fisher Scientific) for 72 hours prior to collection.

\section{Metaphase spreads}

Metaphase spreads were prepared as previously described (45) and then stained for 10 min with Giemsa stain (Ricca Chemical). The slides were blinded prior to counting and at least 50 metaphase spreads were scored per slide per condition.

\section{Whole cell lysate, chromatin fractionation and Western blot analysis}

These techniques were performed, as previously described (16). 


\section{Antibodies and chemical inhibitors}

Primary: SMC1A (Bethyl, A300-055A), SMC3 (Bethyl, A300-060A), acetylated-SMC3 (kindly provided by Dr. Prasad Jallepalli), RAD21 (SCC1) (Bethyl, A300-080A), SCC-112 (PDS5) (Bethyl, A300-089A), OBFC1 (STN1) (Novus, NBP2-01006), CTC1 (46), $\alpha$-tubulin (Sigma, T-9026), TEN1 (47), MAD2 (Bethyl, A300-301A), $\alpha$-Actinin (Santa Cruz, SC17829), H3 (Cell Signaling, 9715), Flag (Thermo Fisher, MA191878, PA1-984B)

Secondary: Thermo Fisher Scientific: anti-rabbit-HRP (Thermo Fisher, 32460), anti-mouse-HRP (Thermo Fisher, 32430), goat anti-rabbit Alexa Fluor 594 (Thermo Fisher, A-11037)

Chemical inhibitors: Cells were treated with aphidicolin (1 $\mu \mathrm{M}$, Millipore-Sigma 178273) or hydroxyurea (2 mM, Millipore-Sigma 400046) for 2 hour or (S)-(+)-camptothecin (1 $\mathrm{MM}$, Millipore-Sigma C9911) for 1 hour.

\section{Live cell imaging}

MSCV-H2B-mRFP1 was created by replacing GFP in MSCV-GFP with the H2B-mRFP1 from pCS-H2BmRFP1, using Notl and Xhol. pCS-H2B-mRFP1 was a gift from Dr. Sean Megason (Addgene \#53745) and MSCV-GFP was a gift from Dr. Tannishtha Reya (Addgene \#20672). HeLa cells were transduced with retrovirus produced in HEK 293T and RFP positive cells were selected through two rounds of fluorescence-activated cell sorting. Approximately 3,000 cells were plated into 96 well IncuCyte ImageLock plates $24 \mathrm{~h}$ prior to imaging. Cells were then imaged at 5 min intervals for $3 \mathrm{~h}$ under a 20x objective, using the IncuCyte S3 Live-Cell Analysis System.

\section{Fluorescent in-situ hybridization (FISH)}

Following collection, cells were washed in $5 \mathrm{ml} 1 \mathrm{x}$ PBS and then fixed by dropwise addition of $5 \mathrm{ml}$ Carnoy's solution (3:1 methanol:acetic acid) with gentle vortexing to avoid clumping. Cells were incubated on ice for $10 \mathrm{~min}$, spun down and supernatant removed. $5 \mathrm{ml}$ of fresh Carnoy's solution was then added and the cells stored at $4^{\circ} \mathrm{C}$. Prior to cytospin, cells were counted and resuspended in fresh Carnoy's solution to a concentration of approximately $500 \mathrm{cells} / \mu \mathrm{l} .100 \mathrm{ul}$ of cell suspension was then spun onto slides at 10,000 rpm for 2 mins using 3-well adaptors (StatSpin Cytofuge 2). Slides were washed with Carnoy's solution and allowed to dry. Chromosome specific FISH was then performed with a Texas Redlabeled chromosome 6 alpha satellite probe, following the manufacturer's protocol (Cytocell).

\section{Immunofluorescence}

Cells were pre-extracted with ice-cold 1x CSK buffer (10 mM HEPES pH 7.4, 0.3 M sucrose, $100 \mathrm{mM}$ $\mathrm{NaCl}, 3 \mathrm{mM} \mathrm{MgCl}_{2}$ ) containing $0.1 \%$ Triton $\mathrm{X}-100$ for $2 \mathrm{~min}$ at room temperature and then fixed with icecold $100 \%$ methanol at $-20^{\circ} \mathrm{C}$ for $10 \mathrm{~min}$. IF was then performed as previously described (16). 
Co-Immunoprecipitation (co-IP)

Co-IP was performed as previously described (16). Plasmids used include pcDNA3.1-Flag-CTC1, pcDNA3.1-Flag-STN1 and pCMV6-TEN1 $(16,18)$.

\section{Proximity Ligation Assay (PLA)}

HeLa 1.2.11 cells were fixed for 20 minutes at room temperature with $4 \%$ formaldehyde in $1 x$ PBS followed by permeabilization with $100 \%$ methanol for 20 min at $-20^{\circ} \mathrm{C}$. Subsequent steps were performed with the Duolink PLA kit (Millipore-Sigma) as previously described (24), except the first wash after primary incubation was performed using wash buffer A, not 5\% BSA in 1x PBS. Primary antibodies: 1:100 mouse $\alpha-S T N 1,1: 600$ rabbit $\alpha-S M C 3$.

\section{Image analysis}

For IF, FISH and PLA, images were taken on an EVOS FL microscope, using a 40x or 60x objective (Thermo Fisher Scientific). At minimum five images were scored per independent, biological experiment for each condition. Image analysis for Figures 1 and 3D was performed using ImageJ and Figures 2D and 3B with CellProfiler.

Supporting information - This article contains supporting information.

Acknowledgements - We would like to thank Yilin Wang, Margaret Pokalsky and Ross Jaeger for assistance with experiments and Dr. Lauren Ball for assistance with the mass spectrometry analysis. We would also like to thank Dr. Christopher Kasbek, Dr. Carolyn Price and the members of the Stewart lab for helpful discussions. Finally, we thank Dr. Carolyn Price (HCT116 CTC1 F/F cells) and Dr. Prasad Jallepalli (Ac-SMC3 antibody) for generously providing cell lines and reagents. Proteomic analysis was performed at the Mass Spectrometry Facility, a University Shared Research Resource at the Medical University of South Carolina, using instrumentation acquired through the NIH shared instrumentation grant program (S10 OD010731).

Funding - This work was supported by funds from the National Institutes of Health (K99 GM104409 and R00 GM104409 to J.A.S.) and startup funds from the University of South Carolina. P.L. was supported in part by a SPARC grant from the University of South Carolina. The content is solely the responsibility of the authors and does not represent the official views of the National Institutes of Health or the University of South Carolina. 
Conflict of interest - The authors declare that they have no conflicts of interest with the contend of this article.

1. Peters, J. M., Tedeschi, A., and Schmitz, J. (2008) The cohesin complex and its roles in chromosome biology. Genes Dev 22, 3089-3114

2. Makrantoni, V., and Marston, A. L. (2018) Cohesin and chromosome segregation. Curr Biol 28, R688-R693

3. Haarhuis, J. H., Elbatsh, A. M., and Rowland, B. D. (2014) Cohesin and its regulation: on the logic of X-shaped chromosomes. Dev Cell 31, 7-18

4. Peters, J. M., and Nishiyama, T. (2012) Sister chromatid cohesion. Cold Spring Harb Perspect Biol 4

5. Uhlmann, F., and Nasmyth, K. (1998) Cohesion between sister chromatids must be established during DNA replication. Curr Biol 8, 1095-1101

6. Wutz, G., Varnai, C., Nagasaka, K., Cisneros, D. A., Stocsits, R. R., Tang, W., Schoenfelder, S., Jessberger, G., Muhar, M., Hossain, M. J., Walther, N., Koch, B., Kueblbeck, M., Ellenberg, J., Zuber, J., Fraser, P., and Peters, J. M. (2017) Topologically associating domains and chromatin loops depend on cohesin and are regulated by CTCF, WAPL, and PDS5 proteins. EMBO J 36, 3573-3599

7. Morales, C., Ruiz-Torres, M., Rodriguez-Acebes, S., Lafarga, V., Rodriguez-Corsino, M., Megias, D., Cisneros, D. A., Peters, J. M., Mendez, J., and Losada, A. (2020) PDS5 proteins are required for proper cohesin dynamics and participate in replication fork protection. J Biol Chem 295, 146157

8. Benedict, B., van Schie, J. J. M., Oostra, A. B., Balk, J. A., Wolthuis, R. M. F., Riele, H. T., and de Lange, J. (2020) WAPL-Dependent Repair of Damaged DNA Replication Forks Underlies Oncogene-Induced Loss of Sister Chromatid Cohesion. Dev Cell 52, 683-698 e687

9. Masamsetti, V. P., Low, R. R. J., Mak, K. S., O'Connor, A., Riffkin, C. D., Lamm, N., Crabbe, L., Karlseder, J., Huang, D. C. S., Hayashi, M. T., and Cesare, A. J. (2019) Replication stress induces mitotic death through parallel pathways regulated by WAPL and telomere deprotection. Nat Commun 10, 4224

10. Stewart, J. A., Wang, Y., Ackerson, S. M., and Schuck, P. L. (2018) Emerging roles of CST in maintaining genome stability and human disease. Front Biosci (Landmark Ed) 23, 1564-1586

11. Lim, C. J., Barbour, A. T., Zaug, A. J., Goodrich, K. J., McKay, A. E., Wuttke, D. S., and Cech, T. R. (2020) The structure of human CST reveals a decameric assembly bound to telomeric DNA. Science 368, 1081-1085

12. Bryan, C., Rice, C., Harkisheimer, M., Schultz, D. C., and Skordalakes, E. (2013) Structure of the human telomeric Stn1-Ten1 capping complex. PLoS One 8, e66756

13. Setiaputra, D., and Durocher, D. (2019) Shieldin - the protector of DNA ends. EMBO Rep 20

14. Lim, C. J., and Cech, T. R. (2021) Shaping human telomeres: from shelterin and CST complexes to telomeric chromatin organization. Nat Rev Mol Cell Biol

15. Zhang, M., Wang, B., Li, T., Liu, R., Xiao, Y., Geng, X., Li, G., Liu, Q., Price, C. M., Liu, Y., and Wang, F. (2019) Mammalian CST averts replication failure by preventing G-quadruplex accumulation. Nucleic Acids Res 47, 5243-5259

16. Wang, Y., Brady, K. S., Caiello, B. P., Ackerson, S. M., and Stewart, J. A. (2019) Human CST suppresses origin licensing and promotes AND-1/Ctf4 chromatin association. Life Sci Alliance 2

17. Chastain, M., Zhou, Q., Shiva, O., Fadri-Moskwik, M., Whitmore, L., Jia, P., Dai, X., Huang, C., Ye, P., and Chai, W. (2016) Human CST Facilitates Genome-wide RAD51 Recruitment to GCRich Repetitive Sequences in Response to Replication Stress. Cell Rep 16, 2048

18. Stewart, J. A., Wang, F., Chaiken, M. F., Kasbek, C., Chastain, P. D., 2nd, Wright, W. E., and Price, C. M. (2012) Human CST promotes telomere duplex replication and general replication restart after fork stalling. EMBO J 31, 3537-3549

19. Ganduri, S., and Lue, N. F. (2017) STN1-POLA2 interaction provides a basis for primase-pol alpha stimulation by human STN1. Nucleic Acids Res 45, 9455-9466 
20. Goulian, M., Heard, C. J., and Grimm, S. L. (1990) Purification and properties of an accessory protein for DNA polymerase alpha/primase. J Biol Chem 265, 13221-13230

21. Hubner, N. C., Wang, L. H., Kaulich, M., Descombes, P., Poser, I., and Nigg, E. A. (2010) Reexamination of siRNA specificity questions role of $\mathrm{PICH}$ and Tao1 in the spindle checkpoint and identifies Mad2 as a sensitive target for small RNAs. Chromosoma 119, 149-165

22. Dynek, J. N., and Smith, S. (2004) Resolution of sister telomere association is required for progression through mitosis. Science 304, 97-100

23. Canudas, S., and Smith, S. (2009) Differential regulation of telomere and centromere cohesion by the Scc3 homologues SA1 and SA2, respectively, in human cells. J Cell Biol 187, 165-173

24. Alam, M. S. (2018) Proximity Ligation Assay (PLA). Curr Protoc Immunol 123, e58

25. Zheng, G., Kanchwala, M., Xing, C., and Yu, H. (2018) MCM2-7-dependent cohesin loading during $S$ phase promotes sister-chromatid cohesion. Elife 7

26. Leylek, T. R., Jeusset, L. M., Lichtensztejn, Z., and McManus, K. J. (2020) Reduced Expression of Genes Regulating Cohesion Induces Chromosome Instability that May Promote Cancer and Impact Patient Outcomes. Sci Rep 10, 592

27. Gerlich, D., Koch, B., Dupeux, F., Peters, J. M., and Ellenberg, J. (2006) Live-cell imaging reveals a stable cohesin-chromatin interaction after but not before DNA replication. Curr Biol 16, 15711578

28. Minamino, M., Tei, S., Negishi, L., Kanemaki, M. T., Yoshimura, A., Sutani, T., Bando, M., and Shirahige, K. (2018) Temporal Regulation of ESCO2 Degradation by the MCM Complex, the CUL4-DDB1-VPRBP Complex, and the Anaphase-Promoting Complex. Curr Biol 28, 2665-2672 e2665

29. Song, J., Lafont, A., Chen, J., Wu, F. M., Shirahige, K., and Rankin, S. (2012) Cohesin acetylation promotes sister chromatid cohesion only in association with the replication machinery. $J$ Biol Chem 287, 34325-34336

30. Terret, M. E., Sherwood, R., Rahman, S., Qin, J., and Jallepalli, P. V. (2009) Cohesin acetylation speeds the replication fork. Nature 462, 231-234

31. Ivanov, M. P., Ladurner, R., Poser, I., Beveridge, R., Rampler, E., Hudecz, O., Novatchkova, M., Heriche, J. K., Wutz, G., van der Lelij, P., Kreidl, E., Hutchins, J. R., Axelsson-Ekker, H., Ellenberg, J., Hyman, A. A., Mechtler, K., and Peters, J. M. (2018) The replicative helicase MCM recruits cohesin acetyltransferase ESCO2 to mediate centromeric sister chromatid cohesion. $E M B O J 37$

32. Megason, S. G. (2009) In toto imaging of embryogenesis with confocal time-lapse microscopy. Methods Mol Biol 546, 317-332

33. Sigoillot, F. D., Huckins, J. F., Li, F., Zhou, X., Wong, S. T., and King, R. W. (2011) A time-series method for automated measurement of changes in mitotic and interphase duration from timelapse movies. PLoS One 6, e25511

34. Silva, R. D., Mirkovic, M., Guilgur, L. G., Rathore, O. S., Martinho, R. G., and Oliveira, R. A. (2018) Absence of the Spindle Assembly Checkpoint Restores Mitotic Fidelity upon Loss of Sister Chromatid Cohesion. Curr Biol 28, 2837-2844 e2833

35. Mirkovic, M., Hutter, L. H., Novak, B., and Oliveira, R. A. (2015) Premature Sister Chromatid Separation Is Poorly Detected by the Spindle Assembly Checkpoint as a Result of System-Level Feedback. Cell Rep 13, 469-478

36. Yoshizawa-Sugata, N., and Masai, H. (2009) Roles of human AND-1 in chromosome transactions in S phase. J Biol Chem 284, 20718-20728

37. Leman, A. R., Noguchi, C., Lee, C. Y., and Noguchi, E. (2010) Human Timeless and Tipin stabilize replication forks and facilitate sister-chromatid cohesion. J Cell Sci 123, 660-670

38. Errico, A., Cosentino, C., Rivera, T., Losada, A., Schwob, E., Hunt, T., and Costanzo, V. (2009) Tipin/Tim1/And1 protein complex promotes Pol alpha chromatin binding and sister chromatid cohesion. EMBO J 28, 3681-3692

39. Chan, R. C., Chan, A., Jeon, M., Wu, T. F., Pasqualone, D., Rougvie, A. E., and Meyer, B. J. (2003) Chromosome cohesion is regulated by a clock gene paralogue TIM-1. Nature 423, $1002-$ 1009 
40. Frattini, C., Villa-Hernandez, S., Pellicano, G., Jossen, R., Katou, Y., Shirahige, K., and Bermejo, R. (2017) Cohesin Ubiquitylation and Mobilization Facilitate Stalled Replication Fork Dynamics. Mol Cell 68, 758-772 e754

41. Tittel-Elmer, M., Lengronne, A., Davidson, M. B., Bacal, J., Francois, P., Hohl, M., Petrini, J. H. J., Pasero, P., and Cobb, J. A. (2012) Cohesin association to replication sites depends on rad50 and promotes fork restart. $\mathrm{Mol}$ Cell 48, 98-108

42. Villa, F., Simon, A. C., Ortiz Bazan, M. A., Kilkenny, M. L., Wirthensohn, D., Wightman, M., MatakVinkovic, D., Pellegrini, L., and Labib, K. (2016) Ctf4 Is a Hub in the Eukaryotic Replisome that Links Multiple CIP-Box Proteins to the CMG Helicase. Mol Cell 63, 385-396

43. Samora, C. P., Saksouk, J., Goswami, P., Wade, B. O., Singleton, M. R., Bates, P. A., Lengronne, A., Costa, A., and Uhlmann, F. (2016) Ctf4 Links DNA Replication with Sister Chromatid Cohesion Establishment by Recruiting the Chl1 Helicase to the Replisome. Mol Cell 63, 371-384

44. Feng, X., Hsu, S. J., Kasbek, C., Chaiken, M., and Price, C. M. (2017) CTC1-mediated C-strand fill-in is an essential step in telomere length maintenance. Nucleic Acids Res 45, 4281-4293

45. Schuck, P. L., and Stewart, J. A. (2019) FISHing for Damage on Metaphase Chromosomes. Methods Mol Biol 1999, 335-347

46. Bhattacharjee, A., Wang, Y., Diao, J., and Price, C. M. (2017) Dynamic DNA binding, junction recognition and G4 melting activity underlie the telomeric and genome-wide roles of human CST. Nucleic Acids Res 45, 12311-12324

47. Kasbek, C., Wang, F., and Price, C. M. (2013) Human TEN1 maintains telomere integrity and functions in genome-wide replication restart. J Biol Chem 288, 30139-30150 


\section{Figure Legends}

Figure 1. CST deficiency results in SCC loss. (A) Western blot analysis of STN1 knockdown and MAD2 levels in HeLa cells. Actinin: loading control. shNT: non-targeting shRNA; shSTN1: shRNA to STN1; and shSTN1 +Flag-STN1: shSTN1-7 cells plus shRNA-resistant Flag-STN1. (B) Example images of metaphase spreads with normal chromosomes or SCC loss. (C) Fold increase in cohesion loss following metaphase spread analysis, as indicated. $n=3$ independent, biological replicates. (D) Representative images of chromosome FISH from cells isolated by mitotic shake-off. Red: centromere 6 probe, Blue: DAPI. Scale bar: $5 \mu \mathrm{m}$ (E) Fold increase in nuclei with $>4$ chromosome 6 foci, indicating SCC loss. $n=4$ independent, biological replicates. (F) Knockdown of CTC1, STN1 or TEN1 by siRNA in HeLa cells. siNT: non-target control, Actinin: loading control. (G) Graph of SCC loss following metaphase spread analysis, as indicated. $n=3$ independent, biological replicates. $P$-values were calculated by an unpaired, two-sided t-test $\left({ }^{\star} P<0.05,{ }^{* \star} \mathrm{P}<0.01,{ }^{* \star *} \mathrm{P}<0.001\right)$.

Figure 2. CST interacts with the cohesin complex. (A) Co-IP of nuclease-treated lysates from HEK 293T cells expressing Flag-tagged CTC1, Flag-tagged STN1 or the full CST complex. (B) Representative images of proximity ligation assay (PLA) performed in HeLa cells with antibodies to STN1 ( $\alpha$-STN1) or SMC3 ( $\alpha$-SMC3) alone or combined ( $\alpha$-STN1/ $\alpha$-SMC3). Red: PLA foci, Blue: DAPI. Scale bar: $5 \mu \mathrm{m}(\mathrm{C})$ Violin plot of PLA foci per nucleus. Results are representative of four independent, biological experiments. Bold dashed line: median, dashed lines: first and third quartiles. $P$-values were calculated by an unpaired, two-sided t-test $\left.{ }^{* * * *} \mathrm{P}<0.0001\right)$.

Figure 3. STN1 depletion does not affect cohesin levels or mitotic timing. (A) Western blot analysis of total SMC and acetylated-SMC3 in HeLa cells, as indicated. (B) Representative images of SMC3 levels in pre-extracted cells. DAPI: blue, SMC3: red. Scale bar: $20 \mu \mathrm{m}(\mathrm{C})$ Western blot of chromatin-bound cohesin subunits. Histone H3 serves as a loading control. (D) HeLa cells stably expressing H2B-RFP were imaged in 5 min intervals for 3 hours. Scale bar: $20 \mu \mathrm{m}(\mathrm{E})$ Dot plot of the time between nuclear envelope breakdown until cytokinesis in individual cells. Black lines and numbers below the graph indicate the average minutes. Error bars indicate the \pm SEM of three independent biological experiments. $\mathrm{n}$ indicates the number of total cells scored. $P$-values were calculated by an unpaired, two-sided t-test $\left({ }^{*} P<0.05,{ }^{* *} P<0.01\right)$.

\section{Figure 4. Replication inhibition increases the CST-cohesin interaction and SCC loss in STN1-} depleted cells. (A) Violin plot of nuclear PLA foci in HeLa cells after treatment with DNA replication inhibitors. Drug treatments were performed for 2 hours for hydroxyurea (HU) and aphidicolin (APH). Camptothecin (CPT) treatment was performed for 1 hour. Bold dashed line: median, dashed lines: first and third quartiles. $n=3$ independent, biological replicates. (B) Cohesion loss in HeLa shNT and shSTN1 
bioRxiv preprint doi: https://doi.org/10.1101/2021.04.11.439363; this version posted April 12, 2021. The copyright holder for this preprint (which

was not certified by peer review) is the author/funder, who has granted bioRxiv a license to display the preprint in perpetuity. It is made available under aCC-BY-NC-ND 4.0 International license.

cells following replication stress. Cells were treated with replication inhibitors as in (A) and then released for 8 hours prior to metaphase spread preparation. Colcemid was added 2 hours prior to collection. $n=3$ independent, biological replicates. $P$-values were calculated by an unpaired, two-sided t-test $\left({ }^{*} \mathrm{P}<0.05\right.$, $\left.{ }^{* *} P<0.01,{ }^{* *} P<0.001\right)$. 
bioRxiv preprint doi: https://doi.org/10.1101/2021.04.11.439363; this version posted April 12, 2021. The copyright holder for this preprint (which was not certified by peer review) is the author/funder, who has granted bioRxiv a license to display the preprint in perpetuity. It is made available under aCC-BY-NC-ND 4.0 International license.

Figure 1

A

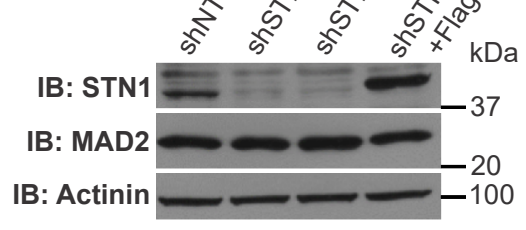

B

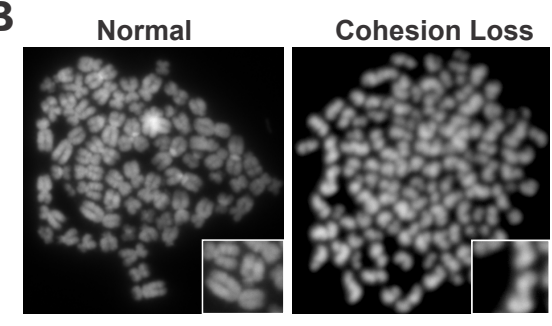

C

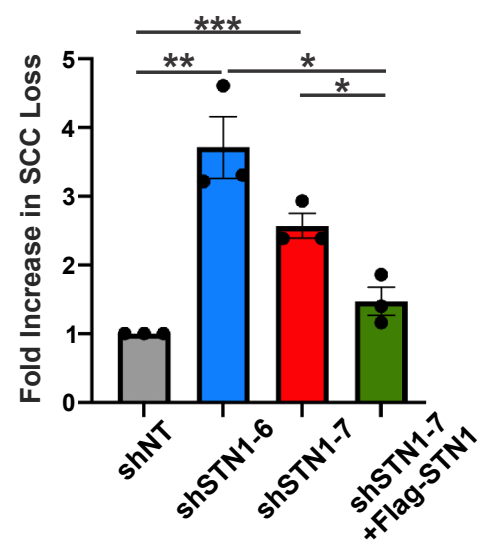

D

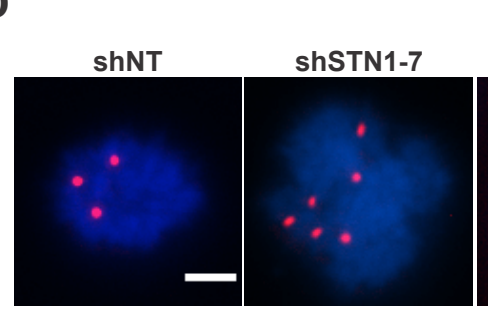

F

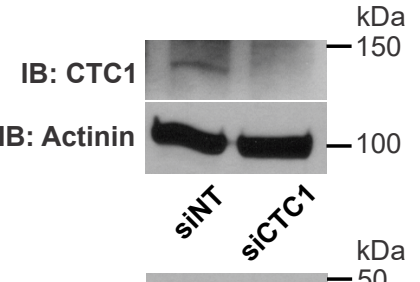

IB: STN1

IB: Actinin

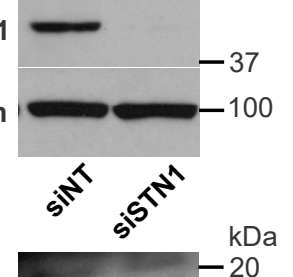

IB: TEN1

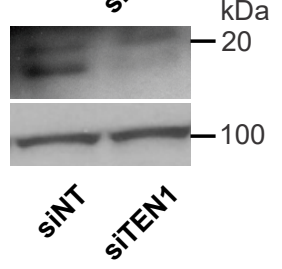

E

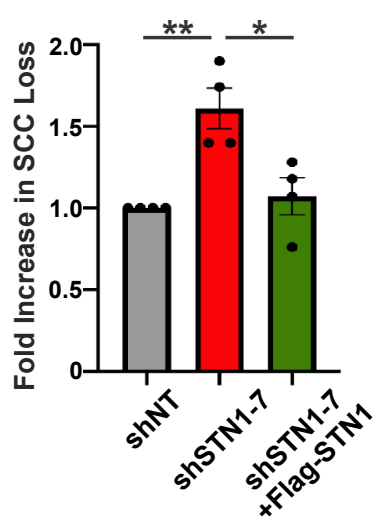

G

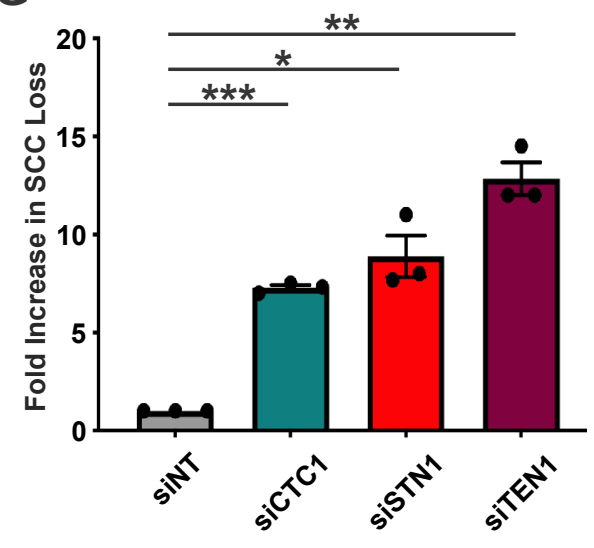


bioRxiv preprint doi: https://doi.org/10.1101/2021.04.11.439363; this version posted April 12, 2021. The copyright holder for this preprint (which was not certified by peer review) is the author/funder, who has granted bioRxiv a license to display the preprint in perpetuity. It is made

Figure 2 available under aCC-BY-NC-ND 4.0 International license.
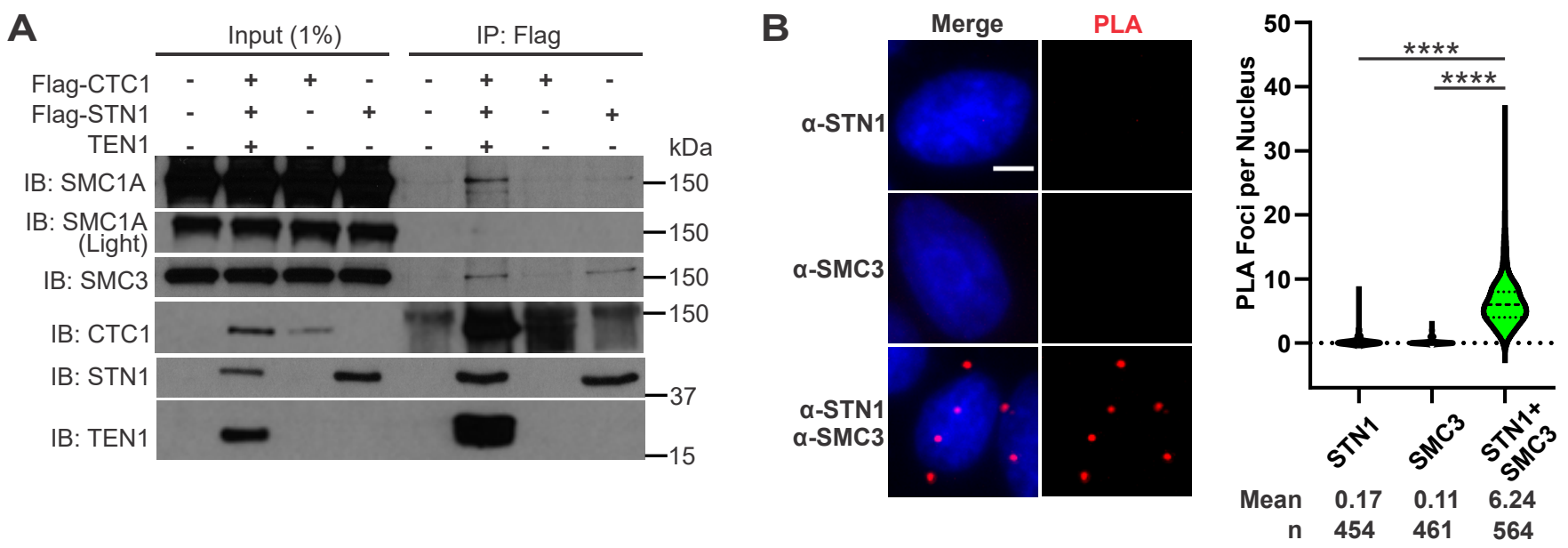
bioRxiv preprint doi: https://doi.org/10.1101/2021.04.11.439363; this version posted April 12, 2021. The copyright holder for this preprint (which was not certified by peer review) is the author/funder, who has granted bioRxiv a license to display the preprint in perpetuity. It is made available under aCC-BY-NC-ND 4.0 International license.

\section{Figure 3}

A

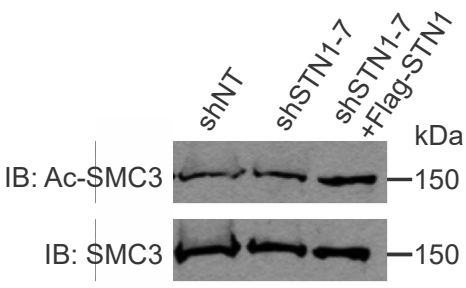

B

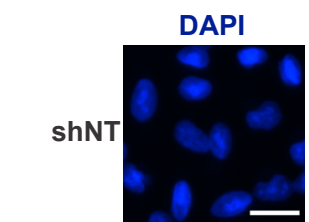

shSTN1-7

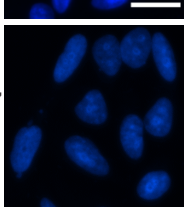

ShSTN1-7 + Flag-STN1

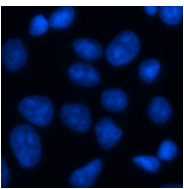

D
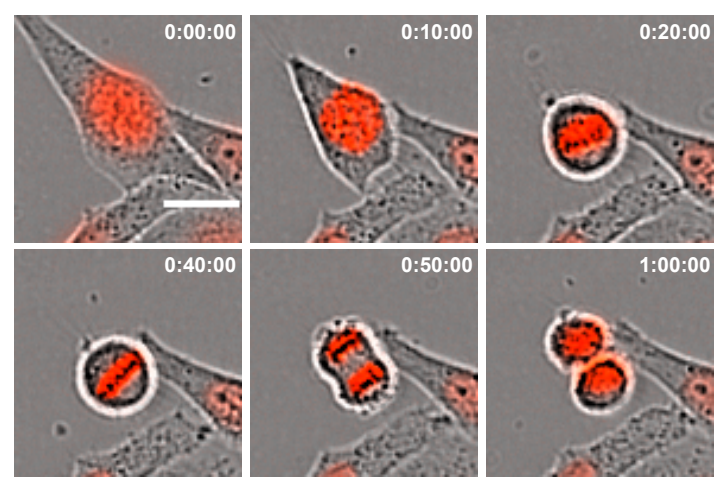

C

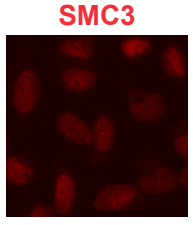

Merge

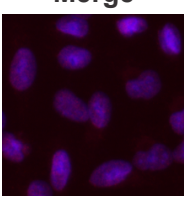

Chromatin के के के के के
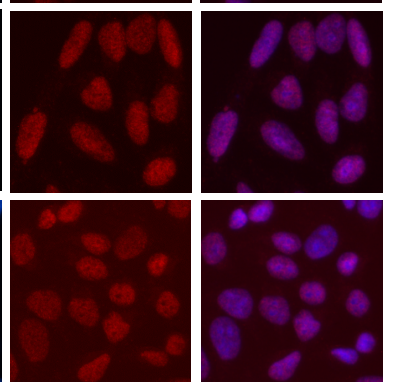

IB: SMC3

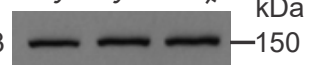

IB: SMC1A

$-150$

IB: RAD21

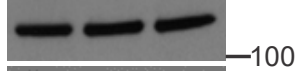

IB: PDS5

IB: H3

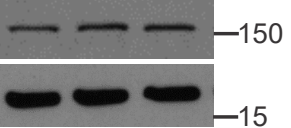

E

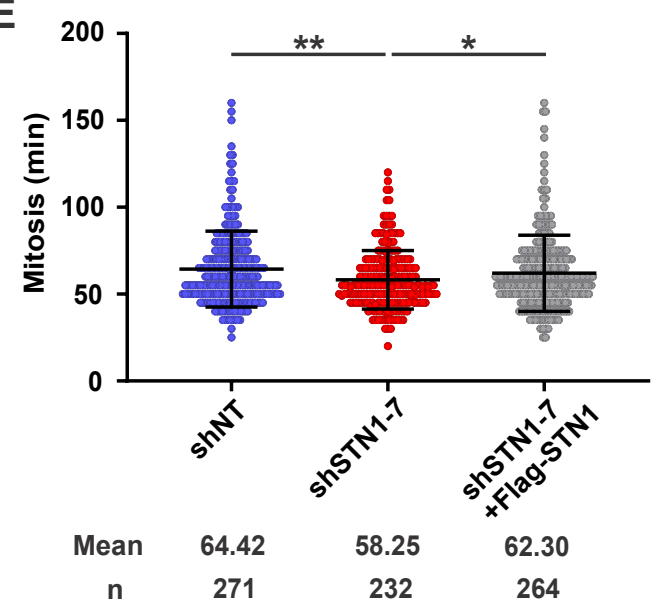


bioRxiv preprint doi: https://doi.org/10.1101/2021.04.11.439363; this version posted April 12, 2021. The copyright holder for this preprint (which was not certified by peer review) is the author/funder, who has granted bioRxiv a license to display the preprint in perpetuity. It is made available under aCC-BY-NC-ND 4.0 International license.

\section{Figure 4}

A

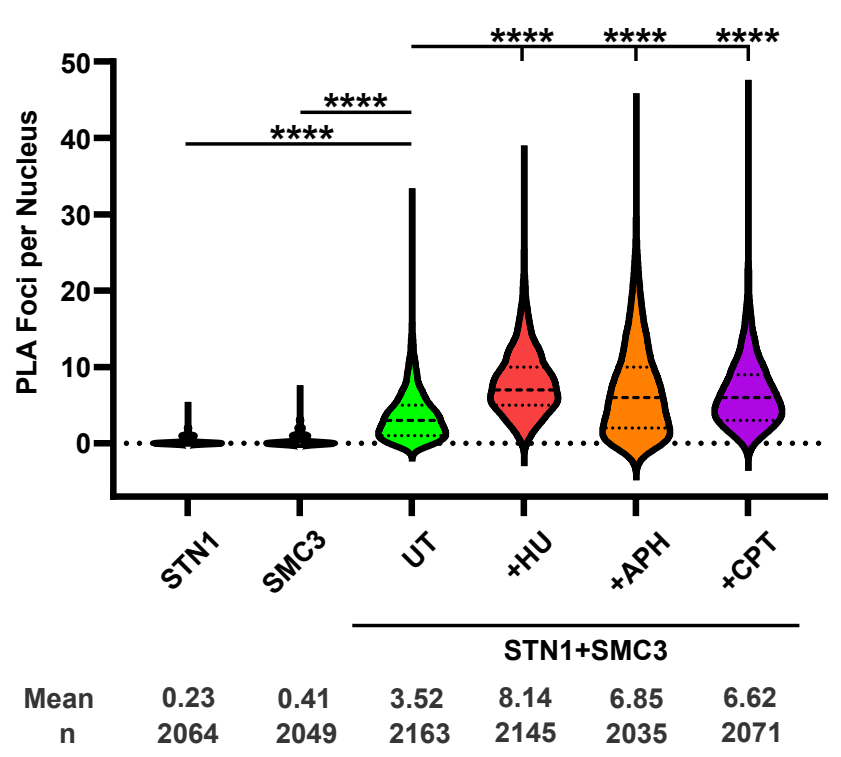

B

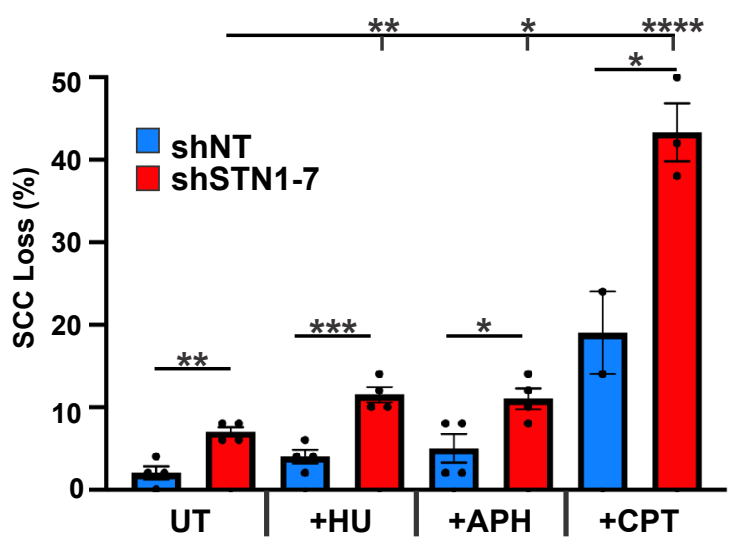

C

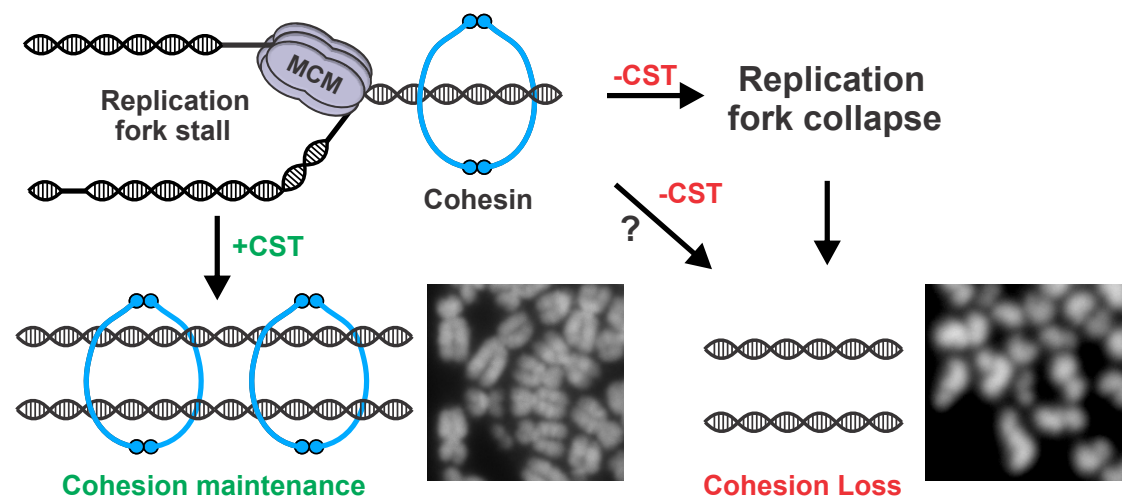

